\section{Research Square}

Preprints are preliminary reports that have not undergone peer review.

They should not be considered conclusive, used to inform clinical practice, or referenced by the media as validated information.

\title{
Impact of spontaneous hepatocellular carcinoma rupture on surgical outcome after liver resection: a systemic review and meta-analysis
}

Wei Zhang ( $\square$ weizhangtih@hust.edu.cn )

Tongji Hospital of Tongji Medical College of Huazhong University of Science and Technology https://orcid.org/0000-0002-3380-1010

Zhi-Yong Huang

Tongji Hospital of Tongji Medical College of Huazhong University of Science and Technology

Chang Shu

Tongji Hospital of Tongji Medical College of Huazhong University of Science and Technology

Research article

Keywords: Hepatocellular carcinoma, Rupture, Prognosis, Liver resection, Meta-analysis

Posted Date: December 17th, 2019

DOI: https://doi.org/10.21203/rs.2.19071/v1

License: (c) (i) This work is licensed under a Creative Commons Attribution 4.0 International License. Read Full License 


\section{Abstract}

Background Spontaneous tumor rupture is a rare but life-threatening complication of hepatocellular carcinoma (HCC). The impact of spontaneous ruptured HCC on long-term survival after liver resection (LR) remains unclear. Our aim was to compare the surgical outcome in patients who underwent LR with ruptured and non-ruptured HCC .

Methods A comprehensive search using PubMed, Embase, Cochrane Library and Science Citation Index Expanded databases was performed. The primary outcomes were the hazard ratio (HR) for overall survival ( OS) and disease free survival (DFS). The secondary outcomes included morbidity, hospital mortality and recurrence rate.

Results Ten retrospective studies including 3222 patients met the inclusion criteria. Pooled analysis revealed a significantly poorer OS and DFS for patients with ruptured $\mathrm{HCC}$ compared to patients with non-ruptured $\mathrm{HCC}(\mathrm{HR}, 2.02 ; 95 \% \mathrm{Cl}, 1.61-2.54 ; \mathrm{P}<0.00001$ and $\mathrm{HR}, 1.92 ; 95 \% \mathrm{Cl}, 1.56$ 2.35; $\mathrm{P}<0.00001$, respectively). In the subgroup analysis, both the propensity score matching (PSM) and non-PSM studies demonstrated a significantly poorer OS in the ruptured HCC group ( $\mathrm{P}=0.02$ and $\mathrm{P}<0.00001$, respectively). However, meta-analysis of two $\mathrm{PSM}$ studies showed that there was not significant difference in the DFS between the two groups $(P=0.50)$. Patients in the ruptured HCC group had a higher mortality and recurrence rate than the non-ruptured $\mathrm{HCC}$ group, but the difference was not significant $(P=0.05$ and $P=0.06$, respectively)

Conclusions Surgical outcomes of the patients with the ruptured or non-ruptured HCC undergoing LR were not only affected by tumor rupture itself, but also by the tumor characteristics and liver functional status. Therefore, classifying all ruptured but resectable HCC as T4 stage is unable to accurately represent their true prognosis.

\section{Background}

Hepatocellular carcinoma (HCC) is the sixth most common malignancy and the second most common cause of cancer related death in men worldwide ${ }^{[1]}$. Spontaneous tumor rupture is a rare but life-threatening complication of HCC. There are distinct geographic variations in the reported incidences. Ruptured HCC occurs in $2.3 \%$ to $26 \%$ of the patients with HCC in Asia and less than $3 \%$ in the West ${ }^{\text {[2] }}$

Ruptured HCC is associated with a high in-hospital mortality rate ranging $25-75 \%$ and poor long-term survival with a median survival of 1.2 - 4 months if untreated ${ }^{[3,4]}$. Therefore, the current TNM classifications of American Joint Committee on Cancer/Union for International Cancer Control (AJCC/UICC) classify ruptured HCC as T4, regardless of other tumor characteristics ${ }^{[5]}$. However, the true impact of spontaneously ruptured HCC on long-term survival and recurrence pattern after liver resection (LR) remains a topic of debate. Several studies have compared the surgical outcomes in patients undergoing LR between ruptured and non-ruptured HCC. The results showed that the survival of patients with ruptured $\mathrm{HCC}$ was significantly worse than that of patients with non-ruptured $\mathrm{HCC}{ }^{[6-8]}$. In contrast, reports from other centers found no significant difference in overall survival (OS) or disease-free survival (DFS) between the two groups of patients ${ }^{\text {[9-14] }}$. A recent study from Hong Kong analyzed the survival outcome stratified according to the TNM classification. The finding showed that the adverse prognostic effect of tumor rupture was mainly limited to early stage tumors and assigning all resectable ruptured tumors to T4 may overestimate the severity of disease ${ }^{[15]}$.

Because of the low incidence of spontaneous HCC rupture and the heterogeneity of patients, the prognostic influence of ruptured HCC was still unclear. There is currently no discussion in the literature that groups the available evidence. We thus performed a systematic review and metaanalysis to compare the surgical outcome in terms of OS and DFS in the patients who underwent LR with ruptured and non-ruptured HCC.

\section{Methods}

\section{Search strategy}

This systematic review was conducted in line with the Preferred Reporting Items for Systematic Reviews and Meta Analyses (PRISMA) ${ }^{[16]}$ and Assessing the Methodological Quality of Systematic Reviews (AMSTAR) guidelines. The search was restricted to the English language and used PubMed, Embase, Cochrane Library and Science Citation Index Expanded (Web of Science) databases in May 2018 without restriction on the publication date. The following keywords using both their MESH terms and free text were used殴liver neoplasms or hepatocellular carcinoma or 'carcinoma, hepatocellular' or hepatoma or liver cell carcinoma or heptocarcinoma or cancer of liver or hepatocellular cancer or 'cancers, hepatocellular' or hepatocellular cancers or hepatic cancer or 'cancer, hepatic' or 'cancers, hepatic' or hepatic cancers or liver cancer or 'cancer, liver' or 'cancers, liver' or liver cancers or cancer of the liver or 'Cancer, Hepatocellular' or hepatic tumor or liver tumor\AND $\bigotimes^{\prime} R u p t u r e$, Spontaneous'or 'Ruptures, Spontaneous' or spontaneous rupture or spontaneous ruptures》 AND \hepatectomy or hepatectomies or liver

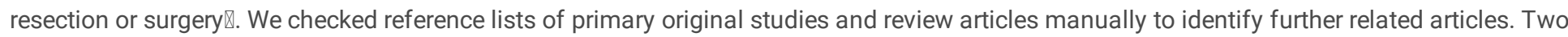
of the authors (WZ, CS) independently carried out a systematic review of the literature. 


\section{Study selection}

The following inclusion criteria were used to select included studies: 1.Original complete publications in English language with full-text accessible; 2.The study included patients diagnosed with ruptured HCC who received liver resection; 3 . The study reported postoperative outcome of liver resection (overall survival and/or disease free survival); 4 . The study compared directly the postoperative outcome according to the presence or absence of spontaneous HCC rupture. Both retrospective and prospective studies were considered eligible. Reviews, case reports, letters, animal or in vitro studies, editorials or expert opinions, and conference abstracts were excluded in the search. Studies published in languages other than English with no translation readily available were also excluded. If dual (or multiple) studies were reported by the same institution and/or authors, either the higher quality publication or more recent publication was included in the analysis. Figure 1 summarizes the process of study identification, inclusion, and exclusion according to PRISMA guidelines.

\section{Data extraction and quality assessment}

Data regarding the following aspects was extracted: inclusion period of study, countries of origin, methodological quality, trial design, duration of follow-up, patient numbers, patient characteristics (age, liver cirrhosis, Child-Pugh class, etiology), tumor characteristics (size, number and vascular invasion), overall survival, disease free survival, morbidity, hospital mortality and recurrence rate. The quality assessment was performed independently by 2 study investigators (WZ, CS), using the Newcastle-Ottawa Quality Assessment Scale (NOS) for observational studies. The maximum total score on this scale is 9 ; studies with scores $\geq 7$ were defined as high-quality studies

\section{Outcome measures}

The primary outcomes evaluated in this meta-analysis were the hazard ratio (HR) for overall survival (OS) and disease-free survival (DFS). OS was defined as the interval from the date of liver resection to the date of death or the last follow-up examination. DFS was defined as the time between the curative resection of HCC and confirmation of recurrence or death. The secondary outcomes included morbidity, hospital mortality and recurrence rate.

\section{Statistical analysis}

The logarithm of the HR with 95\% confidence intervals was used in comparison of the interventions. HR was calculated using the Excel sheet published by Tierney et al. ${ }^{[17]}$, based on the Parmar method ${ }^{[18]}$ of data extraction from survival curves. Statistical heterogeneity was assessed using the $\mathrm{I}^{2}$ test. Heterogeneity was defined as a $\mathrm{P}$ value less than 0.10 or $\mathrm{I}^{2}$ greater than $50 \%$. Sensitivity analysis was conducted by omitting one study by turn to test the robustness of the primary outcomes. Publication bias was assessed by using the funnel plot visually and Egger's regression asymmetry test. We performed preplanned stratified meta-analysis on study-related variables to assess sources of heterogeneity. The following were used as grouping variables for stratified meta-analysis: 1) quality of the studies (low vs. high quality); 2 ) study design (propensity score matching (PSM) vs non-PSM). A p value of 0.05 or less was considered statistically significant. All analyses were performed by using Review Manager, version 5.3 (Nordic Cochrane Centre, Oxford, England) and Stata 12.0 (StataCorp, College Station, Tex).

\section{Results}

\section{Studies included in meta-analysis}

The results of literature search are shown in Fig. 1. Ten retrospective cohort studies published between 1998-2016 were included in the metaanalysis $[6,7,9-14,19,20]$. Three of the studies matched samples by PSM to minimize the effect of potential confounders. Table 1 summarizes the Main characteristics of the included studies. The recruitment period ranged from 1977 to 2013 . Among 10 included studies, 1 was multicenter and 9 single-center. All studies were conducted in East Asia. Five of these ten studies were from Japan, four from China and one from Korea. Ten studies reported the data of postoperative overall survival and seven studies showed the data of postoperative disease-free survival. Each of the studies was independently assessed using the NOS and 7 studies (70\%) were high quality with scores ranging from 7 to 8.

\section{Characteristics of the studied patients}


The total number of patients across all the studies was 3222, of which 377 patients with ruptured HCC and 2845 patients with non-ruptured HCC underwent hepatectomy. Six studies recorded liver cirrhosis. In a fixed model, there was no significant difference between the ruptured and non-ruptured HCC group $\left(\mathrm{I}^{2}=7 \%, \mathrm{OR}=0.84,95 \% \mathrm{Cl} 0.66-1.08, \mathrm{P}=0.12\right)$. Five studies recorded liver function. There was no significant difference in the percentage of Child-Pugh $A$ between the two group $\left(I^{2}=6 \%, O R=1.05,95 \% \mathrm{Cl} 0.68-1.62, \mathrm{P}=0.84\right)$. The percentage of the patients with hepatitis $\mathrm{B}$ infection $(\mathrm{OR}=1.07,95 \% \mathrm{Cl} 0.78-1.46, \mathrm{P}=0.67)$ and with multiple nodules $(\mathrm{OR}=0.93,95 \% \mathrm{Cl} 0.68-1.28, \mathrm{P}=0.67)$ was similar between the two group. Patients in the ruptured HCC group (33.4\%) had a higher prevalence of macrovascular invasion in comparison with patients in the non-ruptured $\mathrm{HCC}$ group $(23.1 \%)$, resulting in an OR of $1.51\left(\mathrm{I}^{2}=40 \%, 95 \% \mathrm{Cl} 1.16-1.96, \mathrm{P}=0.002\right)$.

\section{Overall survival}

Data on OS was available in nine out of the ten studies. The meta-analysis revealed a statistically significant poorer OS for the patients with ruptured HCC compared to the patients with non-ruptured HCC. The pooled HR for OS calculated was $2.02(95 \% \mathrm{Cl} 1.61-2.54, \mathrm{P}<0.00001)$ (Fig.2A). No evidence of publication bias was detected by visual examination of funnel plot (Fig.2B) and with Begg's test (P = 0.466).

There was moderate, borderline significant heterogeneity for this analysis of OS $\left(P=0.05 ; I^{2}=48 \%\right)$. Stratified meta-analysis showed that heterogeneity was largely attributable to the quality of the studies assessed by NOS (test for subgroups difference: $\mathrm{P}=0.007$ ) (Fig.3A). Both studies with high and low quality showed that ruptured HCC was associated to the poorer OS after liver resection, being pooled HR, respectively, of 1.65 (95\% Cl: 1.40-1.95; $\mathrm{P}<0.00001)$ and 2.50 (95\% Cl: 1.94-3.23; $\mathrm{P}<0.00001)$.

Stratified meta-analysis according to PSM analysis showed stable results and no heterogeneity among studies with PSM analysis versus nonPSM (test for subgroups difference: $\mathrm{P}=0.48$ ) (Fig.4A). In the pooled analysis, both the 6 non-PSM studies and 3 PSM studies demonstrated patients in the non-ruptured HCC group had significantly higher OS than the ruptured HCC group $(\mathrm{P}<0.00001$ and $\mathrm{P}=0.02$, respectively). Of note, the studies with PSM analysis provided a lower estimate of the postoperative death risk associated with ruptured HCC, as compared to studies with non-PSM studies $(\mathrm{P}=0.02$ vs $\mathrm{P}<0.00001)$

Sensitivity analysis conducted by excluding each article once per time did not change the main summary estimate.

\section{Disease free survival}

DFS was reported by six studies. The meta-analysis revealed that patients in the ruptured HCC group had a significantly poorer DFS than patients in the non-ruptured HCC group, with a pooled HR of $1.92\left(\mathrm{I}^{2}=22 \%, 95 \% \mathrm{Cl} 1.56-2.35, \mathrm{P}<0.00001\right)$ (Fig.5A). There was not significant heterogeneity in this analysis $\left(P=0.27 ; 1^{2}=22 \%\right)$. No evidence of publication bias was found (Fig.5B).

Stratified meta-analysis according to the quality of the studies showed that ruptured HCC was associated with a poorer DFS after liver resection in both studies with high and low quality. The pooled HR of the different quality studies was $1.91(95 \% \mathrm{Cl}: 1.52-2.40 ; \mathrm{P}<0.00001)$ and 1.94 (95\% Cl: 1.22-3.11; $\mathrm{P}=0.005)$, respectively (Fig.3B).

Stratified meta-analysis according to PSM analysis showed DFS in the ruptured HCC group was significantly poorer than the non-ruptured HCC group in the non-PSM subgroup, with a HR of $2.13\left(\mathrm{I}^{2}=0 \%, 95 \% \mathrm{Cl} 1.70-2.67, \mathrm{P}<0.00001\right)$. On the other hand, meta-analysis of two PSM studies showed that there was not significant difference in the DFS between the two groups. The pooled $\mathrm{HR}$ was $1.18\left(\mathrm{I}^{2}=0 \%, 95 \% \mathrm{Cl} 0.73-1.92\right.$, $\mathrm{P}=0.50$ ) (Fig.4B).

Sensitivity analysis performed by elimination of each article once per time did not show significant deviations in overall summary estimate.

\section{Morbidity, mortality and recurrence rate}

Yang $\mathrm{T}$ et al ${ }^{[6]}$ and Liu CL et al ${ }^{[7]}$ reported similar postoperative morbidity between the two groups. Pooled analysis of these two studies demonstrated the morbidity to be $29.5 \%(n=52 / 176)$ in the ruptured HCC group and $29 \%(n=422 / 1454)$ in the non-ruptured HCC group (OR=1.07, $\left.\mathrm{I}^{2}=18 \%, 95 \% \mathrm{Cl} 0.76-1.51, \mathrm{P}=0.70\right)($ Fig.6A).

Three studies reported hospital mortality. Patients in the ruptured HCC group (5.5\%) had a higher mortality than the non-ruptured HCC group (3.2\%), but the difference was not statistically significant $\left(\mathrm{OR}=1.89, \mathrm{I}^{2}=0 \%, 95 \% \mathrm{Cl} 1.01-3.52, \mathrm{P}=0.05\right)($ Fig.6B). 
Six studies reported postoperative recurrence rate. Among them, Liu CL et al ${ }^{[7]}$ only reported extrahepatic recurrence rate. They found that there was significantly more extrahepatic recurrence in the ruptured group than the non-ruptured group (45.5\% vs $25.8 \%, \mathrm{P}=0.015)$. Pooled analysis of the other five studies demonstrated patients in the ruptured HCC group had a higher recurrence rate than the non-ruptured HCC group (75.8\% vs $60.0 \%)$, however, the difference was not significant ( $\left.\mathrm{OR}=1.76, \mathrm{I}^{2}=55 \%, 95 \% \mathrm{Cl} 0.97-3.2, \mathrm{P}=0.06\right)$ (Fig.6C).

\section{Discussion}

The results of this systematic review and meta-analysis demonstrated a statistically significant poorer OS and DFS for patients with ruptured HCC compared to the patients with non-ruptured HCC. Subgroup analysis was performed based on the study design (PSM or non-PSM). Both the PSM and non-PSM studies demonstrated a significantly poorer OS in the ruptured HCC group. However, meta-analysis of two PSM studies showed that there was not significant difference in the DFS between the two groups. In addition, patients in the ruptured HCC group had a higher mortality and recurrence rate than the non-ruptured HCC group, but the difference was not significant $(P=0.05$ and $P=0.06$, respectively).

Whether HCC rupture affects patient's overall survival and tumor recurrence after liver resection is still unclear and inconsistent from different reports. Liu et al. ${ }^{[7]}$ reported a significantly poorer OS among 33 patients with ruptured HCC who underwent LR than that among the patients with no history of tumor rupture during the same period. Yang T et al. ${ }^{[6]}$ found that the ruptured HCC group had significantly lower OS and recurrence-free survival (RFS) than the non-ruptured HCC group. Other studies have shown similar findings ${ }^{[8,21]}$. However, Yeh $\mathrm{CN}$ et al. ${ }^{[19]}$ reported that patients in the non-ruptured group had a similar overall survival as those in the ruptured HCC group, but significantly better DFS. Studies from other centers also found no significant difference between the two groups of patients in the OS and DFS ${ }^{\text {9-14] }}$. The present metaanalysis has revealed a significantly poorer prognosis in patients with ruptured HCC compared to those with non-ruptured HCC.

Comparing surgical outcomes between spontaneously ruptured HCC and non-ruptured HCC is difficult because discrepancies in patient backgrounds and liver function might significantly influence the prognosis. The ruptures were more frequently observed in patients with liver cirrhosis, a higher AFP concentration, larger tumor, higher tumor stage and poorer liver function and poorly differentiated HCC ${ }^{[8,11,19,21-23]}$. Our pooled results showed that patients in the ruptured HCC group had a higher prevalence of macrovascular invasion in comparison with patients in the non-ruptured HCC group ( $\mathrm{P}=0.002)$. These tumor-related factors (tumor size, location, portal vein tumor thrombosis, and a-fetoprotein level) and host-related factors (the degree of liver damage) have been established as the predictors of long-term survival after LR for patients with HCC ${ }^{[24-26]}$. Ruptured HCC might represent a more advanced tumor stage. These may be the reason why the outcome of ruptured HCC after LR has been poorer than that of non-ruptured HCC.

In order to reduce or eliminate the confounding effects due to the difference in patients' backgrounds, liver function as well as tumor-related factors and clarify the impact of the ruptured HCC on postoperative prognosis, propensity score-matching (PSM) analysis were performed in three recent studies ${ }^{[12-14]}$. After PSM, no statistically significant difference in the OS and DFS was observed between the non-ruptured and ruptured HCC patients. Our subgroup analysis of three PSM studies showed that the patients in the ruptured HCC group had significantly poorer OS than the non-ruptured HCC group. Of note, the studies with PSM analysis provided a lower estimate of the postoperative death risk associated with ruptured HCC, as compared to studies with non-PSM studies ( $P=0.02$ vs $P<0.00001)$. These results demonstrated that in addition to tumor rupture itself, other tumor-related factors and host-related factors can also have a negative impact on patient survival.

One of the mechanisms of rupture has been reported to be venous occlusion by a tumor thrombus, causing an increase in pressure within the tumor [27-29]. Theoretically, tumor rupture can result in early spread of the tumor cells, a higher incidence of eatrahepatic recurrence and poor prognosis. Consistent with these expectations, Miyoshi A et al. ${ }^{[30]}$ found that total recurrence and early recurrence were higher in ruptured HCC group than non-ruptured HCC group. Sada $\mathrm{H}$ et al. ${ }^{[14]}$ reported that ruptured HCC patients had a significantly higher cumulative risk of recurrence than non-ruptured HCC patients. Different from these results, two recently published PSM studies reported that there was no significant difference in the pattern of recurrence between the two groups ${ }^{[12,13]}$. Our subgroup analysis of two PSM studies has shown similar findings.

Rupture itself has been regarded as a worse prognostic factor. All ruptured HCC is defined as T4 in the staging system issued by the AJCC/UICC

${ }^{[5]}$. However, this classification has been questioned. Aoki et al. ${ }^{[22]}$ analyzed the data of 1106 patients with ruptured HCC extracted from the database of a nationwide survey in Japan. The results suggested the TNM stage of spontaneously ruptured HCC corresponded to an additional 0.5-2.0 TNM stage added to the baseline TNM stage. Chan WH et al. ${ }^{[31]}$ retrospectively reviewed 221 patients with T4 HCC. The results indicated that patient with ruptured HCC had better overall survival than those with locally invasive tumors but without ruptures. Of note, the patients who underwent all treatments were included in Aoki's and Chan's studies. Several studies investigated the medical records of the patients who underwent primary hepatecomy for HCC. Yoshida $\mathrm{H}$ et al. ${ }^{[20]}$ found that the cumulative survival rate in the patients with stage 4 disease in the non-ruptured group was lower than that in patients in the ruptured group. Uchiyama et al. ${ }^{[32]}$ reported that the patients with 
ruptured HCC tended to experience better RFS and OS than did the patients with non-ruptured stage IV A. Tumor rupture was neither a risk factor for tumor recurrence nor for OS in multivariate analyses. A retrospective study from Hong Kong [15] showed that tumor rupture was the independent risk factor for poor OS when tumors $<10 \mathrm{~cm}$. When the tumor grew beyond $10 \mathrm{~cm}$, the negative prognostic value of rupture diminished and the poor prognosis would be determined by the other unfavorable tumor features.

This meta-analysis has a number of limitations. The number of studies in the meta-analysis was small. All studies included were retrospective analyses from single centers in Asian. There will have been an inherent selection bias in each of the studies. Due to the difference of HCC etiologies and treatment, the current results may not be generalizable to Western population. The number of patients with ruptured HCC was much smaller than those with non-ruptured HCC and this limits to some extent the precision of the results. Furthermore, clinical heterogeneity with regards to the patient populations remains a major confounding factor.

\section{Conclusion}

To the authors knowledge, this is the first meta-analysis to specifically examine the impact of spontaneous HCC rupture on surgical outcome after liver resection. The results suggested that both OS and DFS of ruptured HCC were inferior to that of non-ruptured HCC in the pooled analysis. Patient's surgical prognosis after liver resection was not only affected by tumor ruptured itself, but also by other tumor-related and host-related factors. Allocation of all ruptured but resectable tumors to T4 stage would overestimate the disease severity and ignore the effects of other tumor biological factors.

\section{Abbreviations}

HCC, hepatocellular carcinoma; AJCC/UICC, American Joint Committee on Cancer/Union for International Cancer Control; LR, liver resection; OS, overall survival; DFS, disease-free survival; PRISMA, Preferred Reporting Items for Systematic Reviews and Meta Analyses; HR, hazard ratio; NOS, Newcastle-Ottawa Quality Assessment Scale; PSM, propensity score matching; RFS, recurrence-free survival; LCSGJ, Liver Cancer Study Group of Japan

\section{Declarations}

\section{Ethics approval and consent to participate}

Not applicable.

\section{Consent to publish}

Not applicable.

\section{Availability of data and materials}

All data generated or analyzed during this study are included in this published article.

\section{Competing interests}

The authors declare that they have no competing interests.

\section{Funding}

This research did not receive any specific grant from funding agencies in the public, commercial, or not-for-profit sectors.

\section{Authors' contributions}

Design of work: WZ; Acquisition, analysis, and interpretation of data: WZ, CS; Drafting and revising: WZ, ZH, CS; Final approval: WZ, ZH; All authors read and approved the final manuscript.

\section{Acknowledgements}

We acknowledge Arian Laurence for critically reading the work.

\section{References}


1. Torre LA, Bray F, Siegel RL, Ferlay J, Lortet-Tieulent J, Jemal A. Global cancer statistics, 2012. CA Cancer J Clin. 2015; 65: 87-108. https://doi.org/10.3322/caac.21262.

2. Yoshida H, Mamada Y, Taniai N, Uchida E. Spontaneous ruptured hepatocellular carcinoma. Hepatol Res. 2016; 46:13-21. https://doi.org/10.1111/hepr.12498.

3. Lai EC, Lau WY. Spontaneous rupture of hepatocellular carcinoma: a systematic review. Arch Surg. 2006; 141: $191-198$. https://doi.org/10.1001/archsurg.141.2.191

4. Al-Mashat FM, Sibiany AM, Kashgari RH, Maimani AA, Al-Radi AO, Balawy IA, Ahmad JE. Spontaneous rupture of hepatocellular carcinoma. Saudi Med J. 2002; 23: 866-870.

5. American Joint Committee on Cancer (AJCC). Cancer Staging Manual, eighth edition. New York: Springer, 2017.

6. Yang T, Sun YF, Zhang J, Lau WY, Lai EC, Lu JH, Shen F, Wu MC. Partial hepatectomy for ruptured hepatocellular carcinoma. Br J Surg. 2013; 100: 1071-1079. https://doi.org/10.1002/bjs.9167.

7. Liu CL, Fan ST, Lo CM, Tso WK, Poon RT, Lam CM, Wong J. Management of spontaneous rupture of hepatocellular carcinoma: single-center experience. J Clin Oncol. 2001; 19: 3725-3732. https://doi.org/10.1200/JCO.2001.19.17.3725.

8. Li J, Huang L, Liu CF, Cao J, Yan JJ, Xu F, Wu MC, Yan YQ. Risk factors and surgical outcomes for spontaneous rupture of BCLC stages A and B hepatocellular carcinoma: a case-control study. World J Gastroenterol. 2014; 20: 9121-9127. https://doi.org/10.3748/wjg.v20.i27.9121.

9. Shuto T, Hirohashi K, Kubo S, Tanaka H, Hamba H, Kubota D, Kinoshita H. Delayed hepatic resection for ruptured hepatocellular carcinoma. Surgery. 1998;124: 33-37.

10. Mizuno S, Yamagiwa K, Ogawa T, Tabata M, Yokoi H, Isaji S, Uemoto S. Are the results of surgical treatment of hepatocellular carcinoma poor if the tumor has spontaneously ruptured? Scand J Gastroenterol. 2004; 39: 567-570. https://doi.org/10.1080/00365520410005135.

11. Zhang XF, Wei T, Liu XM, Lv Y. Spontaneous tumor rupture and surgical prognosis of patients with hepatocellular carcinoma. Scand $\mathrm{J}$ Gastroenterol. 2012; 47: 968-974. https://doi.org/10.3109/00365521. 2012. 685753

12. Lee HS, Choi GH, Kang DR, Han KH, Ahn SH, Kim DY, Park JY, Kim SU, Choi JS. Impact of spontaneous hepatocellular carcinoma rupture on recurrence pattern and long-term surgical outcomes after partial hepatectomy. World J Surg. 2014; 38: $2070-2078$. https://doi.org/10.1007/s00268-014-2502-6

13. Tanaka S, Kaibori M, Ueno M, Wada H, Hirokawa F, Nakai T, lida H, Eguchi H, Hayashi M, Kubo S. Surgical Outcomes for the Ruptured Hepatocellular Carcinoma: Multicenter Analysis with a Case-Controlled Study. J Gastrointest Surg. 2016; 20: $2021-2034$. https://doi.org/10.1007/s11605-016-3280-2.

14. Sada H, Ohira M, Kobayashi T, Tashiro H, Chayama K, Ohdan H. An Analysis of Surgical Treatment for the Spontaneous Rupture of Hepatocellular Carcinoma. Dig Surg. 2016; 33: 43-50. https://doi.org/10.1159/000441531

15. Chan AC, Dai JW, Chok KS, Cheung TT, Lo CM. Prognostic influence of spontaneous tumor rupture on hepatocellular carcinoma after interval hepatectomy. Surgery. 2016; 159:409-417. https://doi.org/ 10.1016/j.surg.2015.07.020

16. Moher D, Liberati A, Tetzlaff J, Altman DG; PRISMA Group. Preferred reporting items for systematic reviews and meta-analyses: the PRISMA statement. Ann Intern Med. 2009;151: 264-269.

17. Tierney JF, Stewart LA, Ghersi D, Burdett S, Sydes MR. Practical methods for incorporating summary time-to-event data into meta-analysis. Trials. 2007; 8:16. https://doi.org/10.1186/1745-6215-8-16.

18. Parmar MK, Torri V, Stewart L. Extracting summary statistics to perform meta-analyses of the published literature for survival endpoints. Stat Med. 1998; 17: 2815-2834.

19. Yeh CN, Lee WC, Jeng LB, Chen MF, Yu MC. Spontaneous tumour rupture and prognosis in patients with hepatocellular carcinoma. Br J Surg. 2002; 89:1125-1129. https://doi.org/10.1046/j.1365-2168.2002. 02188.x

20. Yoshida H, Mamada Y, Taniai N, Mizuguchi Y, Kakinuma D, Ishikawa Y, Kanda T, Matsumoto S, Bando K, Akimaru K, Tajiri T. Long-term results of elective hepatectomy for the treatment of ruptured hepatocellular carcinoma. J Hepatobiliary Pancreat Surg. 2008; 15: $178-182$. https://doi.org/10.1007/ s00534-007-1239-0.

21. Zhu Q, Li J, Yan JJ, Huang L, Wu MC, Yan YQ. Predictors and clinical outcomes for spontaneous rupture of hepatocellular carcinoma. World J Gastroenterol. 2012; 18:7302-7307. https://doi.org/10.3748/wjg.v18. i48. 7302

22. Aoki T, Kokudo N, Matsuyama Y, Izumi N, Ichida T, Kudo M, Ku Y, Sakamoto M, Nakashima O, Matsui O, Makuuchi M; Liver Cancer Study Group of Japan. Prognostic impact of spontaneous tumor rupture in patients with hepatocellular carcinoma: an analysis of 1160 cases from a nationwide survey. Ann Surg. 2014; 259: 532-542. https://doi.org/10.1097/SLA. 0b013e31828846de

23. Moris D, Chakedis J, Sun SH, Spolverato G, Tsilimigras DI, Ntanasis- Stathopoulos I, et al. Management, outcomes, and prognostic factors of ruptured hepatocellular carcinoma: A systematic review. J Surg Oncol. 2018; 117: 341-353. https://doi.org/10.1002/jso.24869. 
24. Hanazaki K, Kajikawa S, Koide N, Adachi W, Amano J. Prognostic factors after hepatic resection for hepatocellular carcinoma with hepatitis C viral infection: Univariate and multivariate analysis. Am J Gastroenterol. 2001; 96: 1243-1250. https://doi.org/10.1111/j.1572-0241. 2001.03634.x

25. Tanaka S, limuro Y, Hirano T, Hai S, Suzumura K, Fujimoto J. Outcomes of hepatic resection for large hepatocellular carcinoma: Special reference to postoperative recurrence. Am Surg. 2015; 81: 64-73.

26. Yip VS, Gomez D, Tan CY, Staettner S, Terlizzo M, Fenwick S, Malik HZ, Ghaneh P, Poston G. Tumour size and differentiation predict survival after liver resection for hepatocellular carcinoma arising from non-cirrhotic and non-fibrotic liver: A case controlled study. Int J Surg. 2013; 11: 1078-1082. https://doi.org/10.1016/j.ijsu.2013.10.001.

27. Ong GB, Chu EPH, Yu FYK, Lee TC. Spontaneous rupture of hepatocellular carcinoma. Br J Surg. 1965; 52: $123-129$.

28. Ong GB, Taw JL. Spontaneous rupture of hepatocellular carcinoma. BMJ. 1972; 4: 146-149.

29. Chearanai O, Plengvanit U, Asavanich C, Damrongsak D, Sindhvananda K, Boonyapisit S. Spontaneous rupture of primary hepatoma: report of 63 cases with particular reference to the pathogenesis and rationale of treatment by hepatic artery ligation. Cancer. 1983; 51: $1532-1536$.

30. Miyoshi A, Kitahara K, Kohya N, Noshiro H, Miyazahi K. Outcomes of patients with spontaneous rupture of hepatocellular carcinoma. Hepatogastroenterology. 2011; 58: 99-102.

31. Chan WH, Hung CF, Pan KT, Lui KW, Huang YT, Lin SY, Lin YY, Wu TH, Yu MC. Impact of spontaneous tumor rupture on prognosis of patients with T4 hepatocellular carcinoma. J Surg Oncol. 2016; 113: 789-795. https://doi.org/10.1002/jso.24245

32. Uchiyama H, Minagawa R, Itoh S, Kajiyama K, Harimoto N, Ikegami T, Yoshizumi T, Shirabe K, Takenaka K, Maehara Y. Favorable Outcomes of Hepatectomy for Ruptured Hepatocellular Carcinoma: Retrospective Analysis of Primary R0-Hepatectomized Patients. Anticancer Res. 2016; 36: 379-385.

\section{Table}

Table 1. Main characteristics of studies included in this systematic review

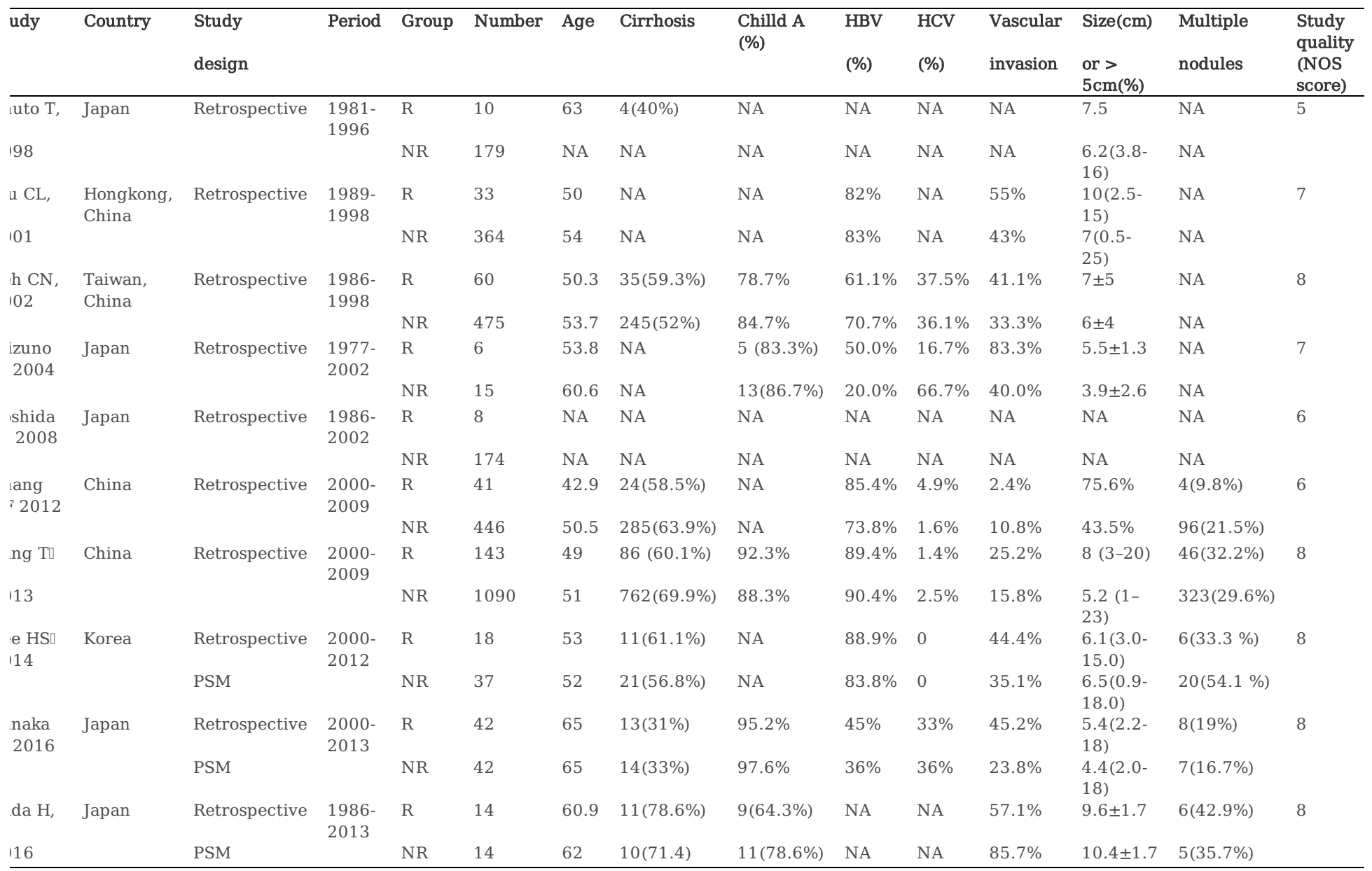

R, ruptured HCC; NR, non-ruptured HCC; HBV, hepatitis B virus-positive; HCV, hepatitis C virus-positive; NOS, NewcastleOttawa Scale; NA, not assessed 


\section{Figures}

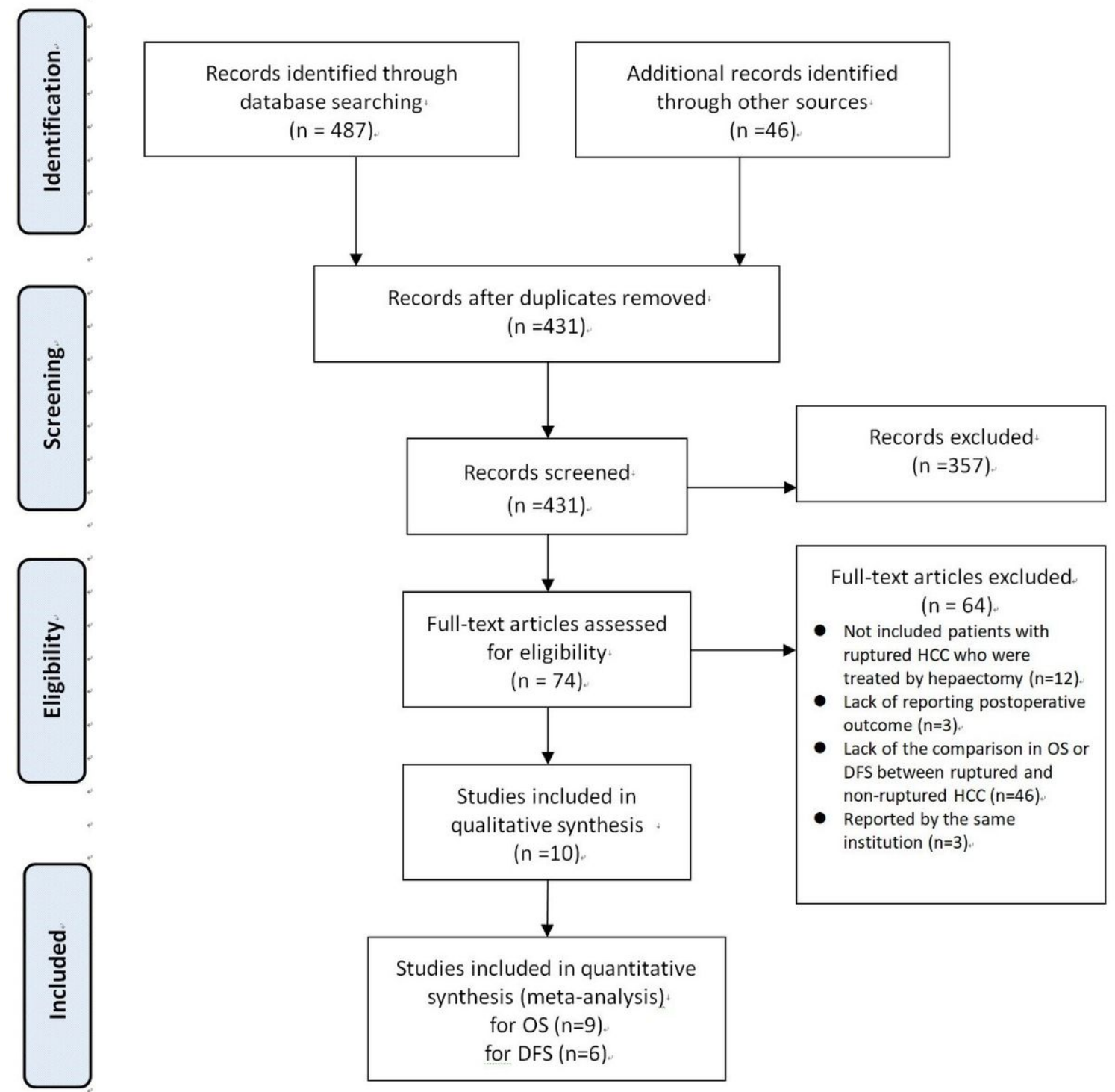

Figure 1

PRISMA Flow chart for selection of studies included in meta-analysis. 
A:

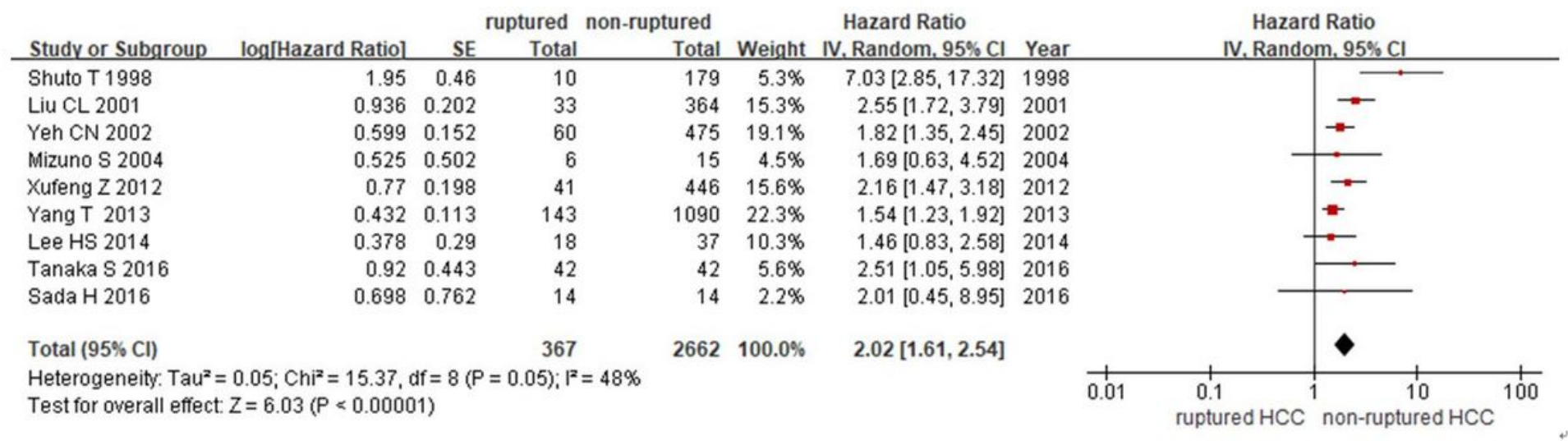

B:

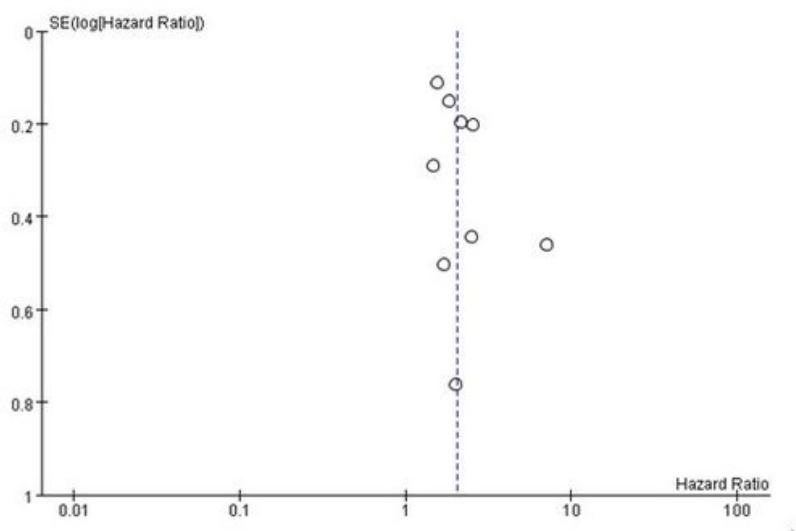

Figure 2

A: Forest plot and B: Funnel plot comparing the overall survival after liver resection between the ruptured HCC group and non-ruptured HCC group. 
A:

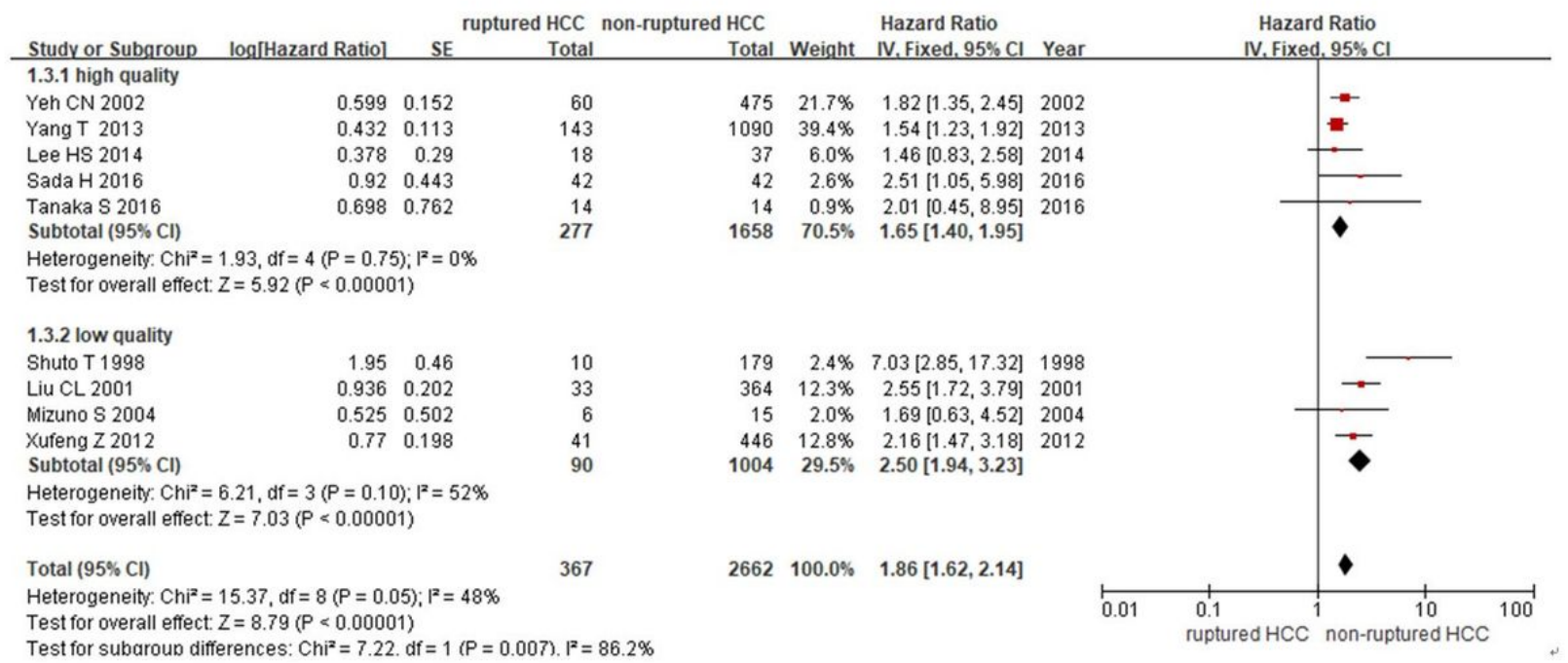

B: .

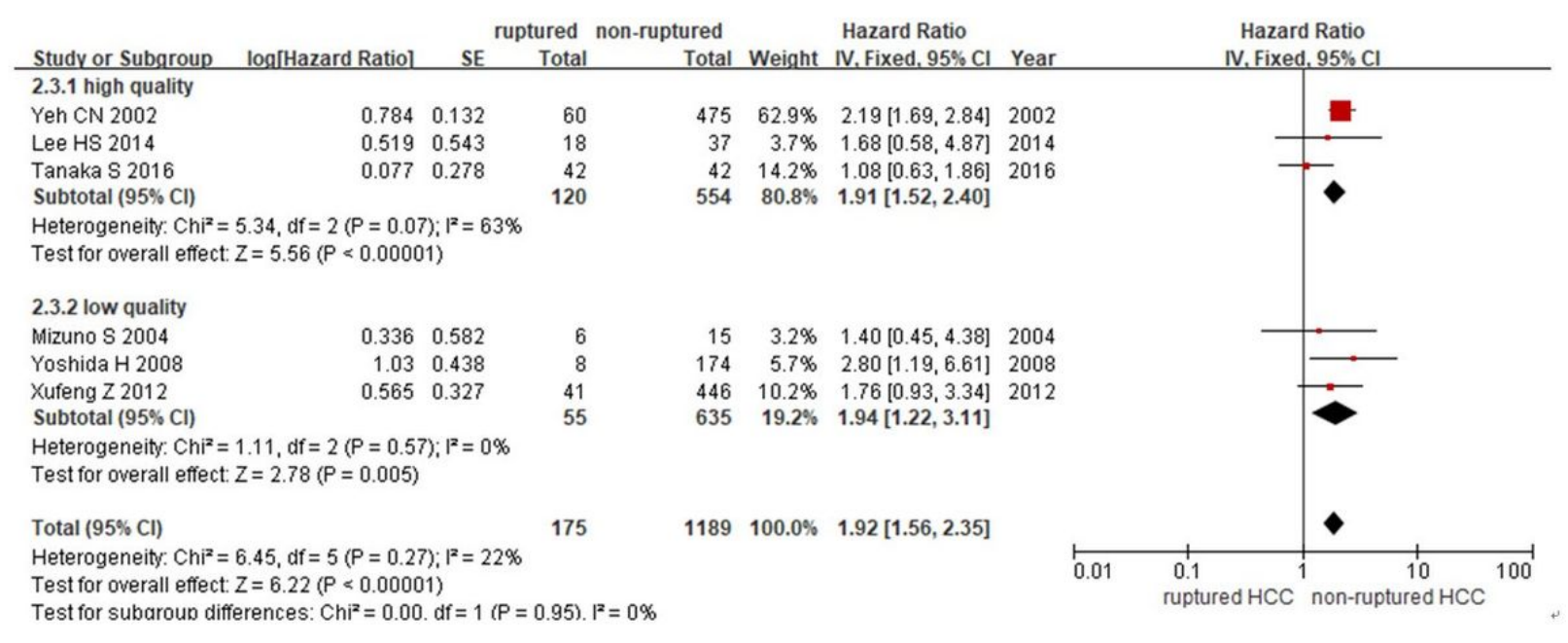

Figure 3

Stratified meta-analysis according to the quality of the studies assessed by NOS (Low vs. High quality). Panel A: Overall survival; Panel B: Disease free survival 
A:

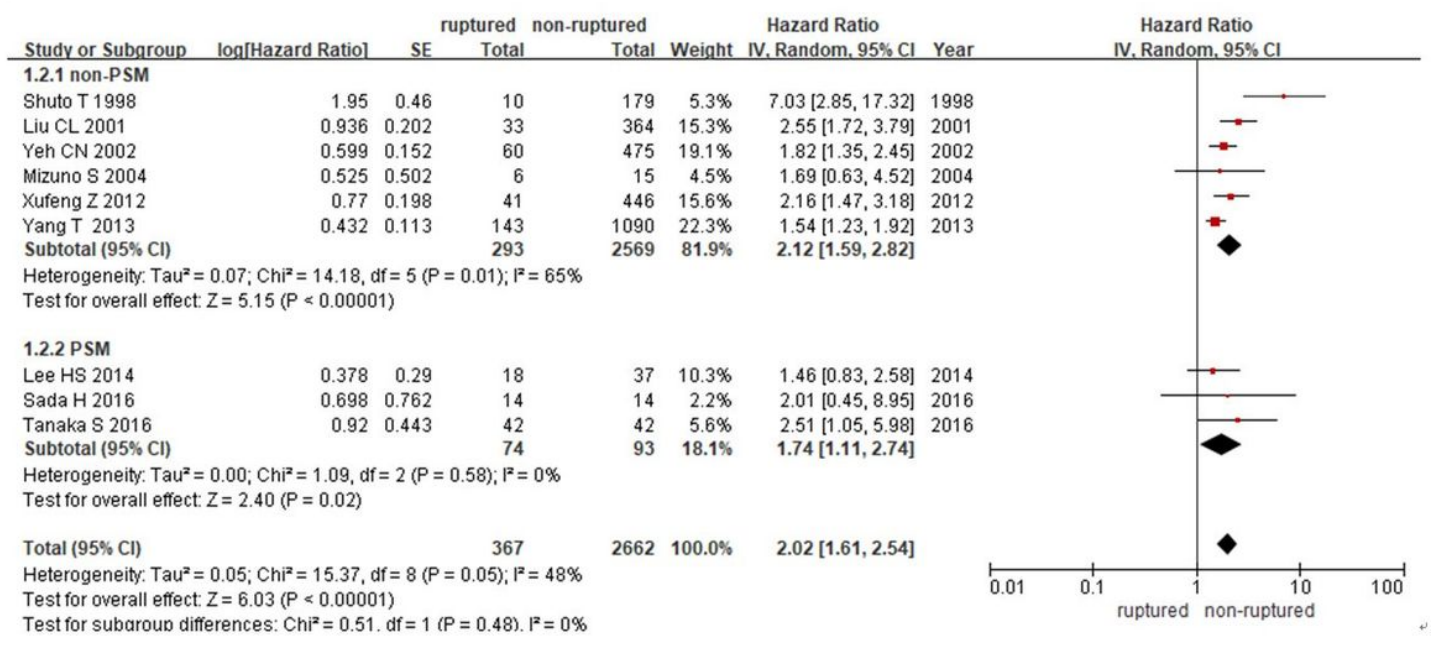

B: .

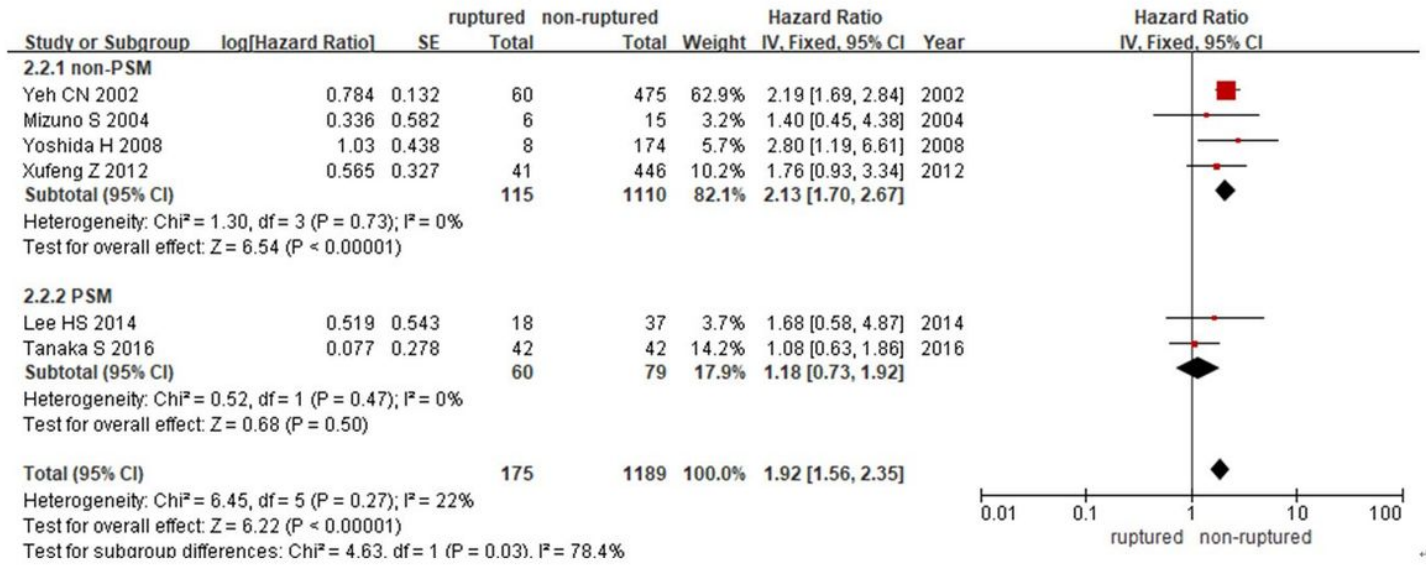

Figure 4

Stratified meta-analysis according to the PSM analysis in the studies (PSM vs. non-PSM). Panel A: Overall survival; Panel B: Disease free survival 
A:

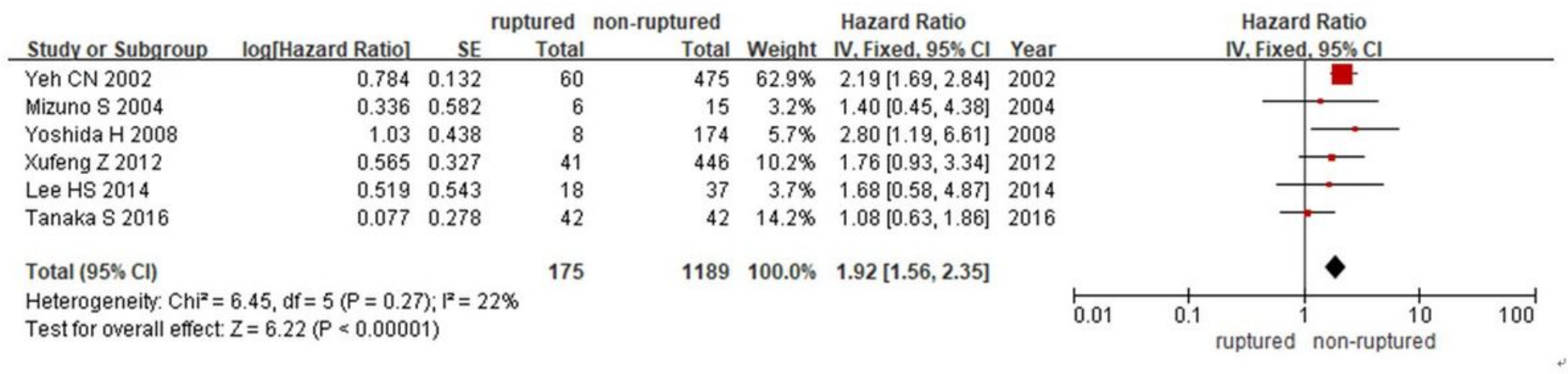

B:

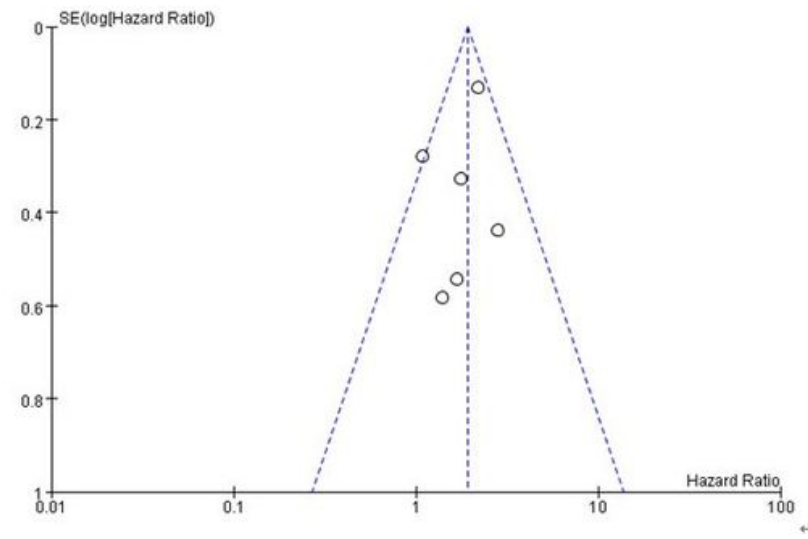

Figure 5

A: Forest plot and B: Funnel plot comparing the disease free survival after liver resection between the ruptured HCC group and non-ruptured HCC group. 
A:

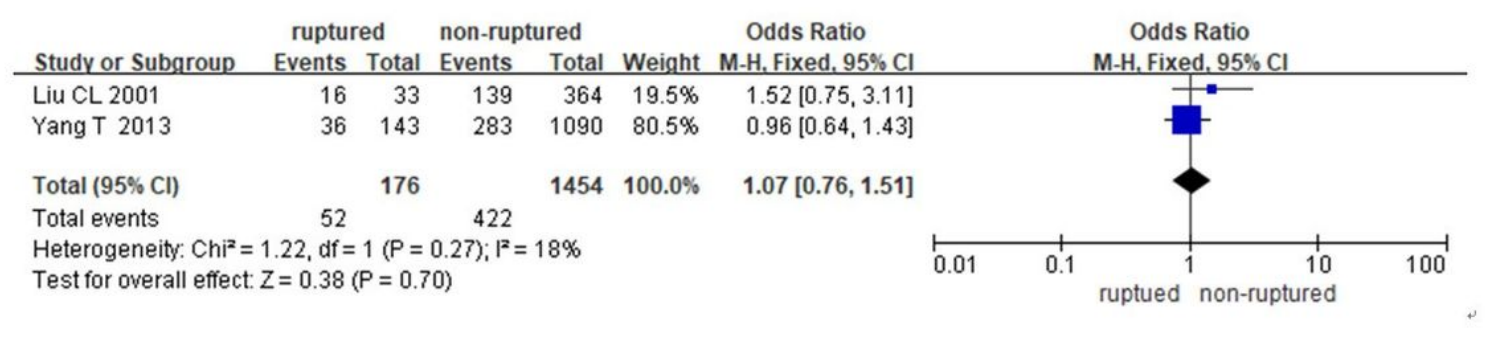

B:

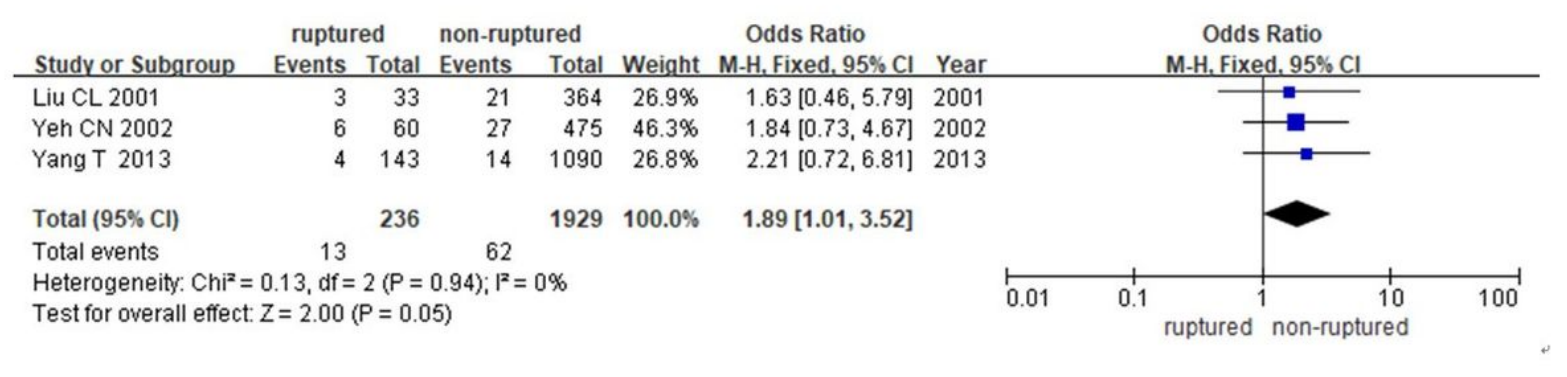

C:

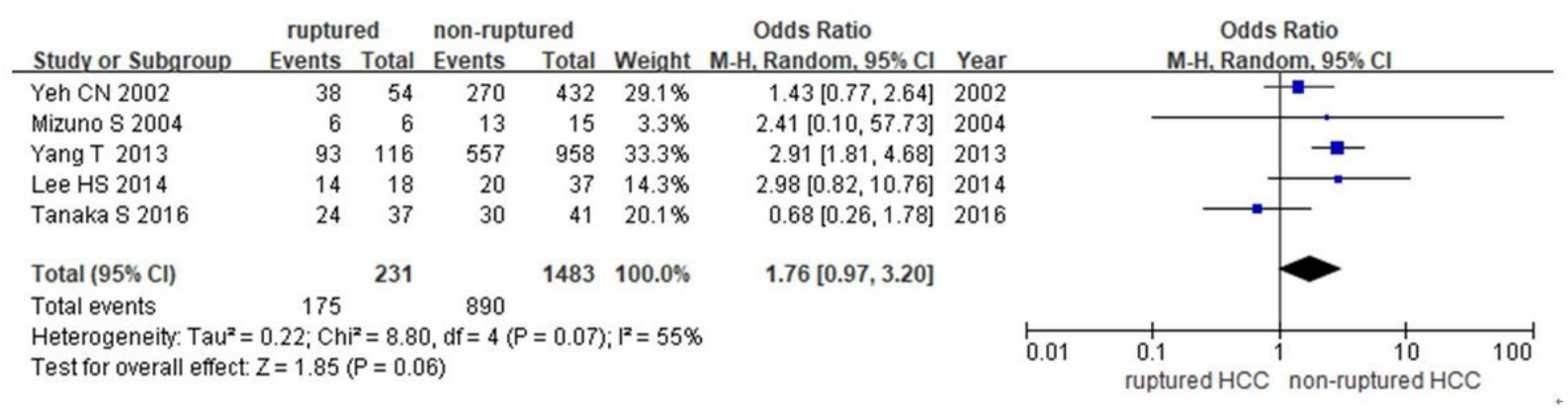

Figure 6

Forest plot comparing A: Morbidity, B: Mortality and C: Recurrence rate after liver resection between the ruptured HCC group and non-ruptured HCC group. 